\title{
Reorienting Immunization Services: Diarrhoea in Vulnerable Population in India
}

\author{
Anil K. Bhola ${ }^{1 *}$, Ananda Amarasinghe ${ }^{2}$ \\ ${ }^{1}$ Independent Public Health Consultant. ${ }^{2}$ Consultant Epidemiologist, Provide complete postal address, Ministry of Health, Sri Lanka.
}

\section{A B STRACT}

A great majority (72\%) of Indian population lives in un-sanitary conditions and nearly $50 \%$ suffers from chronic undernutrition that pre-dispose them to frequent episodes of diarrhoeas of different etiologies. The diarrhoeal outbreaks contributed to $44.9 \%$ of infectious disease outbreaks in 2008 despite significant improvement in safe drinking water supply. The article has a view point that the available vaccines against diarrhoeal diseases should be used in India for vulnerable populations living in settings wherein safe drinking water at point-of-use; and sanitation is still a distant reality. Immunization service should not merely emphasize on prevention of diseases in an isolated manner but it needs to be re-oriented synergistically with other public health interventions leading to health protection, health promotion and subsequent overall health improvement.

Key words: Immunization, diarrhoeas in India, vulnerable population, health protection

\section{BACKGROUND}

Immunization is one of the most successful and costeffective interventions in public health. Use of vaccine has led to the eradication of smallpox, regional elimination of measles and polio, and substantial reductions in the morbidity and mortality attributed to diphtheria, tetanus and pertussis. ${ }^{1}$ The World Health Organization (WHO) estimates that 2 million child deaths were prevented through vaccination in $2003^{2}$ Nevertheless, far more vaccine preventable disease (VPD) deaths could be prevented through optimal use of currently existing vaccines. ${ }^{1,3}$ In India, the burden of six VPDs has been reduced by $85 \%$ since the implementation of the immunization programme in the country. ${ }^{4}$

The diarrhoeal disease is accountable to heavy disease and economic burden in India ${ }^{5,6}$ The annual incidence of all causes-diarrhoeal diseases has increased from 879 (in 2000) to 974 (in 2007) per 100, 000 population, which may be just a tip of the iceberg of diarrhoeal disease burden at population level. ${ }^{5}$ The diarrhoeal disease burden has accounted for $8.2 \%$ of the total disease burden in India. ${ }^{7,8}$ Diarrhoeal diseases are the leading causes of deaths among $1-4$ years $(24 \%)$ and $5-14$ years (17\%) children and accounting

\section{*Address for correspondence:}

E-mail: azadneel@gmail.com

DOI: 10.5530/ijmedph.2.2011.2 for the $5 \%$ of the total deaths in North, West and Southern regions of India from 2001 to $2003 .{ }^{9}$ Out of the total 553 infectious diseases outbreaks reported in 2008 in India, diarrhoeal disease outbreaks accounted for $44.9 \%$, which is the largest group whilst among other non-diarrhoeal diseases the outbreaks of food poisoning $(8.9 \%)$, malaria $(8.3 \%)$, dengue $(7.8 \%)$ and measles $(7.4 \%)$ were the leading causes. ${ }^{10}$ In terms of number of cases reported by the states and Union Territories (UTs) to the Central Bureau of Health Intelligence in 2008, acute diarrhoeal diseases (11.2 million cases) have been only second to the acute respiratory infections with 25.5 million cases. ${ }^{5}$

Diarrhoeal diseases certainly have an economic burden at all levels in India, from household to national. The information on national level economic burden caused by diarrhoeal diseases in India is scarcely available. Median household expenditures per diarrhoeal episode at the referral and the community hospitals in Vellore equaled 5.8\% and $2.2 \%$ of the annual household income, respectively. ${ }^{6}$ The societal cost of a hospitalized diarrhoeal episode among under -5 children in Vellore, Tamil Nadu varied from US $\$ 40.63$ (INR 1648.60) to US $\$ 80.81$ (INR 3278.50) at a large referral hospital and to a smaller community hospital respectively. Considering the diarrhoeal disease burden in India, the amount of cost incurred by it on the health system and on people affected can be thought of; applying the findings from Vellore study to the reported number of all-ages cases of acute diarrhoeal diseases in the year 2007 
at national level, it could be estimated conservatively as about US\$ 446.6 million (INR 18124.1 million), which is $11.9 \%$ of the national budget available for the Ministry of Health and Family Welfare (MOH\&FW), Government of India for 2007-08.5,11

The medical care; health promotion, disease prevention and health protection activities have often been considered as separate, un-linked and even opposing interventions in practice of public health at population level. ${ }^{12,13}$ In the immunization service programme also, it is the disease prevention aspect of the vaccines only which is overemphasized whilst subduing other benefit and opportunities of the programme. ${ }^{13,14}$ There is need to achieve synergy between medical care and public health services. As immunization services have the greatest community penetration, so it should not be merely focusing on reducing infectious disease burden by preventive and medical care services but should also integrate strategies and activities to inculcate the concept of positive well-being and proactive health protection among people.

This paper emphasizes on a timely need of reorienting the immunization services to a new perspective on use of available vaccines as an important adjunctive public health tool with emphasis on health improvement of populations vulnerable to diarrhoeal diseases in India.

\section{VULNERABLE POPULATIONS}

The population with limited or no access to safe drinking water at point-of-use; and to sanitation for whatsoever reasons is vulnerable to diarrhoeal diseases. Such population in India fails to attract adequate attention of the local government bodies, administrators and public health professionals but becomes cynosure of diarrhoeal disease control activities during outbreaks. In urban areas, these vulnerable people are concentrated into most risk-prone areas owing to their individual economic conditions. They lack basic infrastructure and sanitary facilities, often are not prepared for adverse climatic changes and to avoid susceptible environment for diarrhoeal diseases.

The population in India in 2009 was 1.15 billion, which is one sixth of global population..$^{15}$ The population lacking access to sanitation constitutes $72 \%$ of Indian population (47\% urban and $78 \%$ rural). ${ }^{16}$ Although, as per National Family Health Survey-3 (NFHS-3); majority of people ( $96 \%$ urban, $86 \%$ rural) have access to safe drinking water supply; but in reality only $25 \%$ have safe drinking water piped into their house or plot. ${ }^{16,17}$ Thus, remaining $75 \%$ are without safe drinking water in rural areas at point-ofuse; where public taps for drinking water supply is common.
As per 2001 census, only $36.4 \%$ of the total population has latrines within or attached to their houses. In rural areas, the figure is much lower $(21.9 \%)$ wherein open defecation is still prevalent owing to traditional and cultural reasons. ${ }^{16}$

According to a 2005 World Bank estimates, $42 \%$ of Indian population live under the global poverty line of US $\$ 1.25$ a day. ${ }^{18}$ Nearly half of Indian population still suffers from chronic under-nutrition. ${ }^{17,19}$ Almost half of children population (47\%) under-5 years in India are moderate to severely malnourished. ${ }^{17}$ Malnutrition in India is a hidden epidemic; rates for children under-5 varying from highest in Madhya Pradesh (55\%) to Kerala (27\%). ${ }^{17}$ Malnutrition and poverty make people, particularly children, women and elderly more susceptible to diarrhoeal diseases. ${ }^{20,21}$

\section{AVAILABLE INTERVENTIONS}

Launch of the 'National Diarrhoeal Disease Control Programme 1978'; 'Ganga Action Plan 1985', 'National River Conservation Plan 1996' and currently 'Total Sanitation Campaign' have been the major national level efforts in control and prevention of diarrhoeal diseases., ${ }^{716-22}$ The incremental improvement in availability of safe drinking water and sanitation, and better access to treatment has certainly reduced the morbidity and mortality due to diarrhoeal diseases. It was estimated in 1978 that 1.5 million children under- 5 years of age died due to diarrhoea every year, which declined to 0.6-0.7 million in the estimate revised in 1992, supporting the evidence of decline in mortality in general population. ${ }^{7}$ Despite, many mega interventions reducing the burden of diarrhoeal diseases to some extent, still the disease remain continuing in the country as a major public health problem. Acute diarrhoea (40.7\%), cholera $(3.7 \%)$ and typhoid $(1.1 \%)$ are still remaining as leading causes of the total infectious diseases outbreaks in the country. ${ }^{10}$ Thirty five outbreaks of cholera have been reported in four states (West Bengal, Maharashtra, Delhi, Orissa) during the period 1997-2006. ${ }^{23}$

To address the problem of malnutrition, central government mounted national level programmes such as Public Distribution System 1960s' (PDS), 'the Vitamin A Supplementation Programme 1970', 'the Integrated Child Development Services 1975' (ICDS) programme, 'the National Programme of Nutritional Support to Primary Education (NP-NSPE) 1995' and several employment schemes (such as 'the Mahatma Gandhi National Rural Employment Guarantee Act, 2005') providing food and work. However, these programmes and schemes had relatively limited impact on improving nutrition among the poor because of lack of proper implementation. ${ }^{24}$ There 
has been sustained commitment and initiatives to reduce malnutrition but the problem is still huge and continuing. One third of population in India suffering from vitamin and micronutrients deficit and susceptible to diarrhoeal and other diseases. ${ }^{17-21,25}$

\section{Vaccines as a Public Health Intervention}

It is a well established fact that the use of vaccines has demonstrably protected people from contracting diseases and has contributed in promoting health among people living in un-sanitary environment. . $^{2-3,26-30}$ Use of available vaccines for the prevention of diarrhoeal diseases resulting from rotavirus and vibrio cholerae has recently got enhanced attention but yet limited use in India, even as an adjunctive public health tool..$^{4,26-29}$ The traditional belief that vaccines are only for children, is becoming outdated now. The Ministry of Health and Family Welfare, Government of India has already been considering introduction of newer vaccines in India such as rotavirus vaccine. ${ }^{4}$

Although, vaccines give a great hope in reducing the burden of infectious diseases including diarrhoeas still there are some challenges that need attention. The present national immunization programmes are fully stretched with old traditional vaccines and new vaccines; and accommodating newer vaccines within the existing system is a challenge. Increasing programme cost largely incurred by vaccine and supportive logistic cost and additional human resources also necessarily needed to be considered before introduction of any new vaccine, despite the need exists. However, these challenges need to be evaluated beyond the scope of benefits of vaccines on disease prevention only; because it is left silent on other benefits as it has been traditionally thought. ${ }^{4,12-15}$ The available opportunities of the immunization services programme to support and facilitate the delivery of other public health interventions must not be underestimated. In 2004, 73 countries provided Vitamin A with routine immunizations, immunization campaigns or both. ${ }^{2} \mathrm{~A}$ study from Zambia demonstrated a remarkable increase of acceptance and the coverage of insecticide-treated nets (ITNs) when it was integrated within a measles vaccination campaign, with compared to routine isolated programme. ${ }^{31}$ Similarly, in Togo, the family planning service was synergistically linked with Expanded Programme of Immunization (EPI) services resulting 54\% increase in the average monthly number of new family planning clients. ${ }^{32}$ However, the compatibilities between different health interventions at operational level and presence of a strong immunization service prior to such integration is a key to success. ${ }^{33}$ Promoting concepts of health protection and well-beings during vaccination may not only enhance the coverage of immunization but also raise opportunities to effective implementation of other public health interventions in a community. To induce changes, it deserves explicit change in a national vaccine policy or guidelines to highlight the protective and health improvement facilitating aspect of immunization service. Use of vaccines as a public health intervention need change in the attitudes of all stakeholders working in the health sector along with re-orientation of immunization services delivery by promoting synergistic strategies utilizing available public health interventions. It is in tandem with the Global Immunization Vision and Strategies 2006-2015 and the Ottawa Charter., ${ }^{2,34}$

\section{SCOPE}

The scope of reorienting the immunization services appears huge when we think of growing size of vulnerable population to diarrhoeal diseases. Envisioning future: total population of India will exceed 1.3 billion in 2020. ${ }^{22}$ Even if there is no marked growth in the size of the under- 15 population within next decade, still it is constituting 35\% of the total population..$^{22}$ It is reasonable to argue that increased population expansion may also lead to increase in number of slums in urban settings and also may lead to increase in population density even in rural areas. It is unlikely that the provision and availability of safe drinking water and sanitation would meet the needs of the projected population by 2020 . The water and sanitation improvement requires heavy investment. Even with basic infrastructure development, achieving change into healthy behaviour and practices may still remain challenging and may require a longer time. Hence, it will necessitate the adoption of alternative measures to support the vulnerable population.

Use of available vaccines against diarrhoeal diseases in the reoriented immunization services may be worth considering in a timely manner. But, proactive approach of investing in diarrhoeal disease prevention and health protection using licensed vaccines; targeting vulnerable populations only, may also incur additional public health expenditures to the states and requires additional resources. The financial and health sector human resources vary from state to state in India. ${ }^{35}$ There has also been a trend of declining percentage of state resources to the health sector out of the state budget whilst contribution of the central government resources to the overall public health funding has been limited to $15 \%$ only. ${ }^{35}$ The states could introduce new vaccines in public health programme subject to approval by National Regulatory Authority. ${ }^{4}$ But the availability of adequate funds will be a major barrier for use of vaccines as an adjunctive public health tool. Evidence suggested that even with donor support, most developing countries struggle to finance immunization programmes and have been reluctant to introduce the new expensive vaccines. ${ }^{36}$ It is obvious that with the limited financing options, policy makers need to make evidence based decision of new 
interventions, including introduction of new vaccines. However, the benefit of reducing the burden of diarrhoeal disease is needed to be evaluated in broader concepts. The direct impact will be reduction in about $27 \%$ burden of the infectious diseases and significant economic burden on account of diarrhoeal diseases. ${ }^{5,6}$ However, the indirect benefits of avoiding person-time lost due to diarrhoeal diseases will be much larger to the national economy and thus warrants more health economic studies in the field.

To complement the financing of the immunization services against diarrhoeal diseases in India, private sector is an available opportunity. The private sector including community based non-government organizations (NGOs) has huge potential to work in the areas of control and prevention of diarrhoeal diseases including immunization services. The National Health Policy, India 2002 recommends; 'In principle, the state would encourage the handing over of public health service outlets at any level of management for NGOs and other institutions of civil society, on 'as-iswhere-is' basis, along with normative funds for such institutions'. ${ }^{4,35}$ The private sector provides an estimated 10 to $15 \%$ of immunization services. ${ }^{4}$ The public-private mix model may bridge the gap but its sustainability in immunization sector is yet to be tested by population intended to be served. Although, the central government has policy and guidelines for involvement of NGOs and civil society in public health service but the mechanisms to ensure sustainability and accountability are yet to be strengthened, particularly at state level. Immunization services have largely been delivered free-of-cost by the public sector in India so the profits motives of the private sectors also need to be balanced and need to be addressed under a national vaccine policy. Establishment of 'Accredited Immunization Centres' in the private sector may bring innovation in the service delivery methods. The private centres with a capacity of catering to immunization needs of people of all ages; with equitable community access and affordability may be allowed to work after having got accreditation from the health authorities.

The acceptance of primary health care services by the community particularly in rural areas depends on many factors. Positive professional attitudes of health workers and perceived quality service are much important. ${ }^{19,37} \mathrm{~A}$ good public awareness programme on risk-benefits may help in avoiding misconceptions on vaccines and facilitate informed decision making in participation and its acceptance. It further needs to be supported by communication strategies adopted at all levels to make the programme more transparent and closer to the public.

The limited operational and translational research in the public health system is a major constraint in building evidence-based immunization services practice in developing countries, and India has no exception. The main emphasis remains on the statistics of vaccine coverage rather than assessing impact of vaccine effectiveness as a public health intervention in the country. The government decisions on vaccination are increasingly determined by price competition and supply "push" (by the manufacturing companies) rather than "pull" (demand) from proven public health needs. ${ }^{38,39}$ Besides, the lack of scientific evaluations in epidemiological and economic impacts of public health interventions makes it difficult for planning and decision making for other available interventions.

The community health profiling and micro-level health planning is yet to fully utilize the potential of local epidemiology to rationalize the available public health interventions for implementation. It will enable the state and central governments to design context-specific response to diarrhoeal disease outbreaks. The need of enhanced use of epidemiology for better understanding of disease burden, identification of needs and priorities for resource allocation, planning, monitoring and evaluation of health programmes has strongly been recommended in the Delhi Declaration on Epidemiology $2010 .^{40}$ It further emphasizes that it is the responsibility of all stake-holders to assure its full utilization with focus on vulnerable populations. It may enhance and improve use of epidemiological data from local to national level. It will also strengthen the Integrated Disease Surveillance Programme in India.

\section{CONCLUSION}

Providing immune-protection to vulnerable populations along with inculcation of concept of well-being for disease prevention; in adjunct to improvement in water and sanitation environment will accelerate achieving of the Millennium Development Goals (MDG): 1 (target 2): on reduction in poverty (by achieving productive health and abilities for employment among adults), MDG 4: reducing child mortality and MDG 5: reduction in maternal mortality. Although, use of vaccines to reduce the burden of diarrhoeal diseases has its challenges as described in this paper still it is a need of the concurrent time in India that can be phased out gradually with the improvement in availability of safe drinking water at point-of- use and sanitation.

Reorientation of the immunization services for population vulnerable to diarrhoeal diseases is needed with change in perceptions and attitudes at levels of policy formulators, health service providers and people. The use of available vaccines as a public health intervention in conjunction with other available interventions should allow the positive dimensions of health protection and health promotion to 
be highlighted. It will facilitate reducing susceptibility against diarrhoeal diseases in vulnerable populations that live in settings wherein safe drinking water and sanitation improvement is still a distant reality.

\section{REFERENCES}

1. WHO. Weekly Epidemiological Record No. 81, 2006; 189-196.

2. WHO, United Nations Children's Fund. Global Immunization Vision and Strategy 2006-2015, available at http://www.who.int/vaccines/GIVS/ english/GIVS_Final-17Oct05.pdf. Accessed on 7 July 2010.

3. Global Alliance for Vaccine and Immunization. Global Plan of Action for New and Under-utilized Vaccines Implementation: 2008-2011, version 17 December, 2008.

4. MOH\&FW, Government of India. Multi-years Strategic Plan 2005-2010: Universal Immunization Programme, Department of Family Welfare, MOH\&FW, Govt. of India, January, 2005.

5. Central Bureau of Health Intelligence, MOH\&FW. National Health profile, 2008.

6. Mendelsohn AS, Asirvatham JR, Mwamburi DM, Sowmynarayanan TV, Malik V, Muliyil J, Kang G. Estimates of the Burden of Rota-virus-associated and all-cause Diarrohea in Vellore, India. Tropical Med \& Int Health, 2008; 13 (7):934-42.

7. Bhattacharya SK. Progress in the Prevention and Control of Diarrhoeal Diseases since Independence, Natl Med J India, 2003; 16 Suppl 2:15-19.

8. MOH\&FW, Government of India. 'Report of the National Commission on Macroeconomics and Health: Disease Burden in India, Estimation and Causal Analysis', August, 2005.

9. Jha P, Gajalakshmi V, Gupta PC, Kumar R, Mony P. et al. Million Deaths Study (2001-03) Phase I: Final Report, 2009 available at http://cghr.org/ publications.htm. Accessed on 12 July 2010.

10. MOH\&FW, Government of India. Annual Report 2008, Integrated Disease Surveillance Project, 2009.

11. Government of India. Union Expenditure budget 2007-08. Available at http:// indiabudget.nic.in/ub2007-08/eb/sbe46.pdf. Accessed on 7 August 2010.

12. Downie RS., Fyfe C, Tannahil A. Health Promotion: Models and Values, $2^{\text {nd }}$ ed. Oxford University Press, 1996.

13. Tannahill A. Health Promotion: the Tannahill Model Revisited, Public Health, 2009; 123:396-399.

14. Madhavi Y, Puliyel JM, Mathew JL, Raghuram N, Phadke A et al. Evidence based National Vaccine Policy, Indian J. Med. Res. 131, May 2010, pp 617-628.

15. U.S. Census Bureau: International Data Base (IDB), available at http:// www.census.gov/ipc/www/idb/country.php. Accessed on 7 August, 2010.

16. Planning Commission, Government of India. Eleventh Five-Year Plan (2007-2012): Rural Drinking Water and Sanitation in the Eleventh Plan Period-Excerpts, 54 th NDC Meeting; New Delhi, December, 2007.

17. International Institute for Population Sciences (IIPS) and Macro International. National Family Health Survey (NFHS-3), 2005-06: India, Mumbai:IIPS, 2007.

18. World Bank:New Global Poverty Estimates-What it means for India, Available at http://go.worldbank.org/51QB3OCFU0. Accessed on 16 July, 2010.
19. Das MD, Kapoor S, Nikitin D. A closer Look at the Child Mortality among Adivasis in India, Policy Research Working paper 5231, World Bank South Asia Region, Human Development Department, 2010.

20. Khan MA, Yunus M. Host Factors in Childhood Diarrhea. J. Royal Society for the Promotion of Health, 1990; 110 (3):94-95.

21. Gracey M. Diarrhoea and Malnutrition: A Challenge for Paediatricians. J. Pediatric Gastroenterology \& Nutr, 1996; 22 (1):6-16.

22. Planning Commission, Government of India. 'Report of the Committee on India Vision 2020, December, 2002.

23. Kanungo S, Sah BK, Lopez AL, Paiseley A.M., Sur D. et al. Cholera in India: An Analysis of Reports, 1997-2006. Bull. World Health Organ, 2010, 88:185-191.

24. Measham AR, Chatterjee M. (1999): Wasting Away: The Crisis of Malnutrition in India. B. published by World Bank, Washington, D.C.

25. Kotecha PV. Micronutrients Malnutrition in India: Let us say "no" to it now. Indian J. Community Med. 2008; 33 (1):9-10.

26. Clemens J., Holmgren J. Urgent Need of Cholera Vaccines in Public Health Control Programmes, Future Microbiology, Editorial, 2009; 4; 381-385.

27. Lopez A.L., Clemens J.D., Deen J., Jodar L. Cholera Vaccines for the Developing World, Human Vaccines, 2008 4; 165-169.

28. WHO-India. 'WHO Strategic Plan for Strengthening Routine Immunization in India, April 2004.

29. WHO. Weekly Epidemiological Record, No. 13, 26 March 2010; 85:117-128.

30. WHO-SEAR. Department of Communicable Diseases: Profile and Vision, revised; 2007: SEA-CD-145.

31. Grabowsky M, Farrell NT., Hawley WA., Wolkon A, Chimumbwa JM. et al. Integrating Insecticide-treated Bed Nets into a Measles Vaccination Campaign Achieves High, Rapid and Equitable Coverage with Direct and Voucher-based Methods. Trop. Med. \& Intr. Health 2005; 10 (11):1151-1160.

32. Dale H, Aplogan A. The Integration of Family Planning and Childhood Immunization Services in Togo. J. Studies in Family Planning. 1994; 25 (3): $176-183$.

33. Wallace A, Doietz V, Cairns KL. Integration of Immunization Services with other Health Interventions in the Developing World: What works and Why? Systemic literature review. Trop. Med. \& Intern. Health 2008; 14 (1):11-19.

34. Ottawa Charter for Health Promotion, Geneva, World Health Organization, 1986.

35. Government of India. National Health Policy 2002, Available at http:// mohfw.nic.in/NRHM/Documents/National_Health_policy_2002.pdf, Accessed on 15 July, 2010.

36. Wenger JD, DiFabio L, Landaverde JM, Levine OS, Gaafar T. Introduction of HiB Conjugate Vaccines in the Non-Industrialized World: Experience in Four 'Newly Adopting Countries'. Vaccine, 1999; 18:736-742.

37. Patil AV, Somasundaram KV, Goyal RC. Current Health Scenario in Rural India. Australian J. of Rural Health, 2002; 10 (2):129-135.

38. Madhavi Y. The Manufacturer of Consent: Hepatitis B Vaccination, Economic and Political Weekly, June 14, 2003.

39. Madhavi Y. Vaccine Policy in India, PLoS Med. May, 2005; 2 (5):e127.

40. WHO-SEAR. Delhi Declaration on Epidemiology, South-East Asia Regional Conference on Epidemiology, 8-10 March, 2010, New Delhi. 